\title{
Cationic Solid Lipid Nanoparticles Loaded by Cysteine Proteinase Genes as a Novel Anti-Leishmaniasis DNA Vaccine Delivery System: Characterization and In Vitro Evaluations
}

\author{
Delaram Doroud $^{1,2}$, AlirezaVatanara ${ }^{1}$, Farnaz Zahedifard ${ }^{2}$, Elham Gholami ${ }^{2}$, Rouhollah Vahabpour ${ }^{3}$, Abdolhossein \\ Rouholamini Najafabadi ${ }^{1}$ and Sima Rafati ${ }^{2}$ \\ ${ }^{1}$ Department of Pharmaceutics, School of Pharmacy, Tehran University of Medical Sciences, Tehran, Iran
${ }^{2}$ Molecular Immunology and Vaccine Research Laboratory, Pasteur Institute of Iran, Tehran, Iran
${ }^{3}$ Hepatitis and AIDS Department, Pasteur Institute of Iran, Tehran, Iran
}

Received, January 3, 2010; Revised, July 28, 2010; Accepted, August 27, 2010; Published, September 5, 2010

\begin{abstract}
Purpose. Leishmaniasis is a major health problem in many tropical and sub-tropical countries and development of a safe and easily-available vaccine has high priority. Although several antigens potentially capable of inducing protective immunity have been studied, in the absence of pharmaceutical industry interest they have remained as fine publications only. Amongst them, Cathepsin L-like cysteine proteinases (CPs) have received considerable attention and type I and II CPs have been used in a heterologous prime-boost vaccination regime for experimental visceral leishmaniasis in dogs. Due to the promising results of the mentioned vaccination regime, we aimed to evaluate cationic solid lipid nanoparticles (cSLNs) for in vitro delivery of cpa, $c p b$ and $c p b^{\mathrm{CTE}}$ intended to be used as a cocktail DNA vaccine in our forthcoming studies. Methods: cSLNs were formulated of cetyl palmitate, cholesterol, DOTAP and Tween 80 via melt emulsification method followed by high shear homogenization. Different formulations were prepared by anchoring pDNAs on the surface of cSLNs via charge interaction. The formulations were characterized according to their size and zeta potential as well as pDNA integrity and stability against DNase I treatment. Lipoplexes' cytotoxicity was investigated on COS-7 cells by MTT test. The effect of the DOTAP:pDNA ratio on protection ability and cytotoxicity was also studied. In vitro transfection efficiency was qualified by florescent microscopy and quantified using flow cytometry technique. Results: cSLN-pDNA complexes were formulated with suitable size and zeta potential. Efficiency/cytotoxicity ratio of cSLN-pDNAs formulations was comparable to linear PEI-25kDa-pDNAs polyplexes while exhibiting significantly lower cytotoxicity. Conclusion: Tested formulations were able to deliver immunogenic CP genes efficiently. This data proves the ability of this system as a promising DNA vaccine carrier for leishmaniasis to cover the main drawback of naked pDNA delivery that is rapidly elimination from the circulation.
\end{abstract}

\section{INTRODUCTION}

Leishmaniasis is a disease caused by intracellular protozoan parasites of the genus Leishmania, with a diverse set of zoonotic diseases in which humans are incidentally infected. The disease is currently endemic in 88 countries, some of which are among the poorest in the world, affecting 12 million people and threatening 350 million people worldwide (1). Multiple species of Leishmania are known to cause human diseases. Amongst them, cutaneous leishmaniasis (CL) due to Leishmania major ( $L$. major) infection tends to form lesions in the skin with duration typically from few months to a year. Currently, no effective vaccine against leishmaniasis is available and controlling the disease is based on two main strategies, both not efficient enough: controlling Leishmania vectors and reservoir containments, and long-term administration of a limited number of toxic drugs that have partial effectiveness, sometimes accompanied by severe side effects and drug resistency (2).

\footnotetext{
Corresponding Authors: Sima Rafati, Molecular Immunology and Vaccine Research Laboratory, Pasteur Institute of Iran, Tehran, Iran, s_rafati@yahoo.com; Abdolhossein Rouholamini Najafabadi Department of Pharmaceutics, School of Pharmacy, Tehran University of Medical Sciences, Tehran, Iran, Roholami@sina.tums.ac.ir.
} 
Therefore, much attention has been given toward development of effective vaccines. Among different vaccination strategies, DNA vaccination is a potent activator of innate and adaptive immunity for such an intracellular pathogen. It induces both Th1 response and $\mathrm{CD}^{+}$cytotoxic $\mathrm{T}$ lymphocytes, accompanied by producing correctly-folded polypeptides with long period of expression and an inherent adjuvant capacity in the form of $\mathrm{CpG}$ motifs $(3,4)$. DNA vaccines are also more potent and safer than live attenuated and killed vaccines with no adverse reactions in the injection site, enabling simultaneous administration of multiple plasmids without interference.

Searching for protective antigens, as recombinant proteins or DNA vaccines, has been a main objective of many investigations. Unfortunately, they induce only partial protection and/or need some clinically unacceptable adjuvants to induce proper Th1 response in humans (5). Among them, cysteine proteinases (CPs) are enzymes that belong to the papain super family, which are found in a number of organisms from prokaryotes to mammals. In Leishmania, extensive studies have shown that CPs are involved in parasite survival, replication and the onset of disease, which makes them attractive vaccine and/or drug targets for the control of leishmaniasis (6). Previously, our research team has shown that the native form of L. major CPs are recognized by lymphocytes taken from human and mice infected with Leishmania. Immunization experiments using CPs type I (CPB) and type II (CPA) have been carried out in mouse model as well as outbreed dogs with different strategies including DNA and recombinant protein vaccination $(7,8)$. Recently, prime-boost vaccination using $\mathrm{C}$-terminal extension (CTE) of $L$. infantum CPs type I have been performed in BALB/c mice and exhibited both type 1 and 2 immune responses. We showed that although CTE is highly immunogenic, it could direct the immune response toward the Th2 response (9). Therefore, there was a need for further investigating and examining the protective potential of the mentioned cocktail vaccine including $\mathrm{cpb}$ without CTE fragment $\left(\mathrm{cpb}^{-\mathrm{CTE}}\right)$.

Despite all of these valuable data, it is crucial to emphasize that although DNA vaccination offers promises against experimental leishmaniasis, these vaccines are known to be poorly immunogenic and still faced with a number of significant hurdles. It is worth mentioning that DNA immunization is relatively a new strategy and DNA vaccine delivery is still an immature field and no delivery system has yet proven to be sufficiently effective in vivo. However, this field has been improving rapidly in the recent years. The major obstacle in DNA delivery is transporting the DNA into the nucleus of target cells, where they express the foreign gene, before its degradation. In this regard, several pharmaceutical approaches are introduced to overcome these drawbacks. An appropriate vaccine formulation would offer good opportunities to increase vaccine efficacy. In this concern, various colloidal carrier systems have been extensively studied for the formulation of DNA vaccines. Among them, lipid-based delivery systems for DNA in vivo administration represent one of the most advanced delivery technologies to date. They protect loaded DNA while improving its pharmacokinetic characteristics and enhance DNA intracellular uptake and delivery to target APCs (10). Cationic solid-lipid nanoparticles (cSLN) are one of the colloidal lipid-based delivery systems which consist of physiologically well-tolerated ingredients mostly already approved for pharmaceutical application in humans, (11). The solid matrix can protect active biologic ingredients (i.e. DNA) against degradation and allows modulation of the release profiles (10). SLN can be produced without utilizing toxic solvents and even large scale production is possible (11). Furthermore, SLNs with suitable composition have been shown to be well tolerated in vitro, as well as after in vivo administration in mice and rats $(12,13)$. Therefore, Solid Lipid Nanoparticles (SLN) are offered to be a promising alternative to the other colloidal delivery systems.

In the present study, cSLNs were prepared by a melt emulsification method followed by High Shear Homogenization (HSH) method. Plasmids containing CPs type I and II were anchored on the cationic surface of nanoparticles via charge interactions to produce a cSLN-based cysteine proteinases delivery system. Various cSLN-pDNA formulationes were prepared as a function of weight ratio between cationic lipid and pDNAs and characterized by measuring size and surface charge values. The cytotoxicity and gene expression effect of cSLN-pDNAs was evaluated and compared to linear PEI (25-kDa)-pDNA polyplexes as a standard control. 
Since 2001 when Solid Lipid Nanoparticles were launched as non-viral transfection systems, very few reports on their application for gene delivery and no comprehensive report about their usage in anti parasitic DNA vaccination have been published. Here in this paper, we evaluate the incorporation of immunogenic $\mathrm{CP}$ genes into these non-toxic nanoparticles as a novel non-viral DNA vaccine formulation against leishmaniasis.

\section{MATERIALS AND METHODS}

\section{Materials}

Cetylpalmitate, Tween 80, sodium cholate and cholesterol were purchased from Merck (Germany). $N$-[1-(2,3-Dioleoyloxy) propyl]- $N, N, N-$ trimethylammonium chloride (DOTAP), deoxyribonuclease I (DNase I), sodium dodecyl sulfate (SDS), and MTT [3-(4,5-dimethylthiazol-2yl)-2,5-diphenyltetrazolium bromide] were purchased from Sigma-Aldrich (Germany). pEGFP-N1 encoding the green fluorescent protein (GFP) was purchased from Bioscience Clontech. Linear PEI (MW=25 kDa) was purchased from Polyscience (Europe). The materials employed for agarose gel electrophoresis were acquired from Bio$\mathrm{Rad}$ and those applied for PCR and enzymatic digestion were acquired from Roche Applied Sciences. Cell culture reagents including Fetal Calf Sera (FCS), trypsin, EDTA and RPMI were purchased from GIBCO (Germany). To avoid surface-active impurities, autoclaved $\mathrm{MilliQ}^{\mathrm{TM}}$ water was used in all the experiments.

\section{Plasmid Construction and Purification}

pEGFP- ppb $^{-C T E}$

$C p b^{-C T E}$ gene was amplified from the construct pGEM-cpb (8), using the following sense and anti sense primers including $\left(5^{\prime}\right.$ and $3^{\prime}$ BamHI and HindIII restriction sites are underlined, respectively) H6KB: 5' - CGGGATCCACCAT GGATGCGGTGGACTGGCGCG - 3'; and CpbE: 5' - CTGAAGCTTCACATGCG CGGACACGGG-3'. The PCR product $(650 \mathrm{bps})$ was digested with BamHI and HindIII, gel purified and subcloned into pcDNA 3.1(-). Plasmid DNA was purified from recombinant clones (QIAGEN Plasmid Midi Kit, Germany), and verified by restriction enzyme digestion and sequencing using the dideoxy chain termination method on an automated sequencer. After confirmation, the desired gene was sub-cloned into pEGFP-N1 for transfection and named as pEGFP- $c p b^{-C T E}$.

\section{pEGFP-cpa and pEGFP-cpb}

Both pGEM-cpa and pGEM-cpb were available from previous studies (8). Therefore, both $c p a$ and $c p b$ fragments from these constructs were cloned into pEGFP-N1. In all three resulted constructs (pEGFP- $c p a$, pEGFP- $c p b$, and pEGFP- $c p b^{-C T E}$ ), the $c p a, c p b$, and $c p b^{-C T E}$ open reading frames were under control of the CMV promoter, inserted downstream of a Kozak consensus sequence and in frame with an initiation codon. Plasmid DNAs were transformed into the DH5 $\alpha E$. coli strain and purified by alkaline lysis method (QIAGEN Plasmid Midi Kit) and then confirmed by PCR and digestion (data not shown). The total concentration and purity of DNA was determined by NanoDrop ${ }^{\circledR}$ ND-1000 spectrophotometer (Labtech, UK).

\section{Preparation of cSLN formulations}

Application of cetylpalmitate and Tween 80 for SLN formulation was reported elsewhere $(14,15$, 16). We used a slightly modified method to prepare SLN formulation while different process variables were evaluated in an experimental design matrix consisted of 9 runs $\left(3^{2}\right)$ which were obtained by Design-Expert 6.0.6 statistical software. The preparation factors investigated here were the surfactant:total lipid ratio and the preparation method that is either based on melt emulsification (ME), HSH, or a combination of both methods, i.e homogenization of the obtained hot oil in water mixture before cooling $(\mathrm{M}+\mathrm{H})$. The effect of these variable factors were evaluated on the size, zeta and stability of the formulations.

To determine required amount of DOTAP to form a stable complex with pDNA, a 200- $\mu 1$ reaction mixtures were prepared by adding increasing amounts of DOTAP dissolved in milli $\mathrm{Q}^{\mathrm{TM}}$ water to $5 \mu \mathrm{g}$ of pDNA and incubated for 60 min at room temperature. A gel retardation assay was then applied for monitoring the pDNA/DOTAP complex formation by loading $15 \mu \mathrm{l}(0.37 \mu \mathrm{g} / \mu \mathrm{l}$ DNA) of samples onto a $0.8 \%$ agarose gel using 0.5 $\mu \mathrm{g} / \mu \mathrm{l}$ ethidium bromide.

For preparing SLN formulations based on experimental design matrix sodium cholate was added to the hot water phase instead of desired amount of DOTAP that was prescreened in the 
previouse step. The lipid phase contained a constant amount of cetylpalmitate and cholesterol (core and helper lipids) for all the runs. The entire full factorial design of experiment is listed in Table 1.

Table 1. Variable factors applied in the design of experiment for SLN formulation

\begin{tabular}{lll}
\hline Formulation code & $\begin{array}{l}\text { Total lipid ratio: } \\
\text { Tween 80 }\end{array}$ & $\begin{array}{l}\text { Formulation } \\
\text { method }\end{array}$ \\
\hline $\mathrm{S}_{1}$ & 1 & $\mathrm{HSH}$ \\
$\mathrm{S}_{2}$ & 1 & $\mathrm{ME}$ \\
$\mathrm{S}_{3}$ & 1 & $\mathrm{M}+\mathrm{H}$ \\
$\mathrm{S}_{4}$ & 2.5 & $\mathrm{HSH}$ \\
$\mathrm{S}_{5}$ & 2.5 & $\mathrm{ME}$ \\
$\mathrm{S}_{6}$ & 2.5 & $\mathrm{M}+\mathrm{H}$ \\
$\mathrm{S}_{7}$ & 4 & $\mathrm{HSH}$ \\
$\mathrm{S}_{8}$ & 4 & $\mathrm{ME}$ \\
$\mathrm{S}_{9}$ & 4 & $\mathrm{M}+\mathrm{H}$ \\
\hline
\end{tabular}

In order to prepare the final cationic formulation, briefly desired amount of DOTAP $(0.4 \% \mathrm{w} / \mathrm{v})$ was dissolved in hot aqueous phase. The hot aqueous phase was then added to the melted cetyl palmitate and cholesterol $(5.1 \% \mathrm{w} / \mathrm{v})$ phase containing Tween 80 as a nonionic surfactant at the ratio obtained from the experimental design and emulsification was carried out by stirring the mixture at $2000 \mathrm{rpm}$ by a mechanical stirrer (IKA ${ }^{\mathrm{R}}$, Germany) for 10 min at $90{ }^{\circ} \mathrm{C}$. Samples were then homogenized using a high shear homogenizer (IKA ${ }^{\mathrm{R}}$, Germany) at $18,000 \mathrm{rpm}$ for $15 \mathrm{~min}$. cSLN dispersion was obtained by direct cooling of hot $\mathrm{O} / \mathrm{W}$ microemulsion on an ice-bath while stirring at 1000 rpm. Reproducibility of the results was also studied. cSLNs were washed by centrifugation $(6000 \mathrm{rpm}$, $10 \mathrm{~min}$, three times) using the Amicon ${ }^{\circledR}$ Ultra centrifugal filters (Millipore) to purify the suspension from excess surfactants.

\section{pDNA Loading on Nanoparticles}

\section{Preparation of cSLN-pDNA complexes}

Volumes corresponded to $5 \mu \mathrm{g}$ of each purified pEGFP plasmid DNA (pEGFP-cpa, pEGFP-cpb, pEGFP- $c p b^{-C T E}$ and vector without insert as control) were mixed with different amounts of cSLN suspensions in Milli- $\mathrm{Q}^{\mathrm{TM}}$ water separately. The amounts of cSLNs required for complete DNA binding were determined individually by agarose gel electrophoresis and expressed as $\mathrm{w} / \mathrm{w}$ ratio of DOTAP/pDNA. DOTAP/pDNA ratios assayed ranged from 10:1 to 1:1. cSLN-pDNA complexes were prepared by adding pDNA solution to cSLN suspension and $60 \mathrm{~min}$ incubation at room temperature.

\section{Preparation of PEI-pDNA complexes}

PEI-pDNA complexes were prepared by mixing appropriate volumes of $10 \mu \mathrm{M}$ linear PEI $(25 \mathrm{kDa})$ solution (calculated for $7,10,13 \mathrm{~N} / \mathrm{P}$ ratios) with $5 \mu \mathrm{g}$ of each pEGFP-N1construct (pEGFP-cpa, pEGFP- $c p b$, pEGFP- $c p b^{-C T E}$ and vector without insert as control) and incubating the mixture at room temperature for $60 \mathrm{~min}$.

\section{Assessment of Nanoparticles}

\section{Measurement of Size and zeta potential}

The sizes of cSLNs and cSLN-pDNA complexes were determined by photon correlation spectroscopy (PCS). For this purpose, the SLN in water dispersions were diluted in the ratio of 1:100 before analysis. Both size and zeta potential measurements were performed on a Malvern Zetasizer 3000 (Malvern Instruments, Worcestershire, UK). The analysis was performed on each sample immediately after preparation of the cSLNS and $2 \mathrm{hrs}$ after preparing the cSLN-pDNA complex in milli $\mathrm{Q}$ water.

\section{Gel retardation analysis}

Gel retardation assay was applied to determine complex formation between pDNA $(5 \mu \mathrm{g})$ and cSLNs (DOTAP different weight ratios). For this purpose, $15 \mu \mathrm{l}$ aliquots of the complexes samples (containing $0.375 \mu \mathrm{g}$ of pDNA) were subjected to electrophoresis on a $0.8 \%$ agarose gel containing $0.5 \mu \mathrm{g} / \mu \mathrm{l}$ ethidium bromide after mixing with $7 \mu \mathrm{l}$ loading buffer $(0.25 \%$ bromophenol blue and $30 \%$ glycerol). Electrophoresis was carried out in $80 \mathrm{~V}$ for about $2 \mathrm{~h}$ in $1 \mathrm{X}$ TBE running buffer. The bands were observed with a transilluminator (Bio Doc$\mathrm{IT}^{\mathrm{TM}}$ System, UK). Images were captured using a digital camera.

\section{DNase I protection study}

In order to assess protection ability of cSLNs for loaded pDNAs, DNase I was added to cSLNpDNA complexes to a final concentration of $0.8 \mathrm{U}$ DNase I to $5 \mu \mathrm{g}$ DNA and the mixtures were 
incubated at $37^{\circ} \mathrm{C}$ for $1 \mathrm{hr}$. Then SDS solution was added to the samples to a final concentration of $1 \%$ to release DNA from cSLNs. Samples were then analyzed by electrophoresis on agarose gel $0.8 \%$ containing ethidium bromide and the integrity of the DNA in each sample was visualized and compared with free DNA as control.

\section{Stability studies}

The physicochemical stability of cSLNs alone and in complex with pDNA constructs were evaluated at $4 \pm 1^{\circ} \mathrm{C}, 25 \pm 1^{\circ} \mathrm{C}$ at dark for 1 month at regular time intervals via observation of any changes in suspension clarity, particle size and zeta potential assessments.

\section{In Vitro Studies}

\section{Cytotoxicity assay}

COS -7 cells (ATCC no. CRL 1654) were plated at a density of $1 \times 10^{4}$ cells/well in a 96 well microtiter plates at $37^{\circ} \mathrm{C}$ in $5 \% \mathrm{CO}_{2}$ in RPMI as growth medium and incubated at $37{ }^{\circ} \mathrm{C}$ overnight in $5 \%$ $\mathrm{CO}_{2}$ atmosphere (Memmert, USA). Then the medium was removed and cells were washed with serum-free RPMI medium and $200 \mu 1$ of serum-free RPMI medium was added to each well. After overnight incubation, SLN suspensions or PEI solution sample in desired concentrations were added to the medium and cells were incubated for another $24 \mathrm{hrs}$. As controls, a well with only medium (100\% viability) and a well with $2 \%$ triton $\mathrm{X}(0 \%$ viability) were assigned. The plate was incubated for $24 \mathrm{hrs}$ in $37^{\circ} \mathrm{C}$. Then $100 \mu \mathrm{l} \mathrm{MTT}$ solution per $1 \mathrm{ml}$ medium was added to each well after removing the media. Four hrs after incubation, the insoluble formazan crystals were solubilzed in DMSO and absorption was measured at $570 \mathrm{~nm}$ in an automated ELISA plate reader (Bio-Tek ELx808, UK). Viability was expressed in percent compared to untreated cells (100\% survival). Experiments were performed in triplicates and repeated at least twice. Relative cell viability was calculated by dividing [Abs] (mean absorbance) of treated cells to $[\mathrm{Abs}]$ of the control cells.

\section{Plasmid Delivery Assays}

\section{Transfection studies via fluorescent microscopy}

COS-7 cells were transfected with pEGFP-N1 (encoding enhanced GFP as a positive transfection control), pEGFP- $c p a$, pEGFP- $c p b$ and pEGFP- $c p b^{-}$ ${ }^{C T E}$ using SLN and linear PEI $(25 \mathrm{kDa})$. Nontransfected COS-7 cells were used as negative control.

Briefly, the day prior to transfection, $1.5 \times 10^{5}$ COS-7 cells were seeded in each well of 4-well plates (SPL life sciences, South Korea) and grown in RPMI 5\% supplemented with heat-inactivated FCS until the cell reached $75 \%$ confluency. Cell culture medium was replaced with serum free medium immediately before transfection. PEIpDNA and cSLN-pDNA complexes were added to the cells and incubated for $6 \mathrm{hrs}$ at $37{ }^{\circ} \mathrm{C}$ in $5 \%$ $\mathrm{CO}_{2}$, and then the medium was replaced with fresh complete RPMI containing 5\% FCS. The GFP expression of positive transfection control (the COS-7 cells transfected by pEGFP-N1) was confirmed by an inverted fluorescence microscopy (Nikon E200, USA). Observations and image captures were performed using a $10 \mathrm{X}$ objective at 24, 48 and $72 \mathrm{hrs}$ after transfection. The expression of $L$. major $\mathrm{CPA}, \mathrm{CPB}$ and $\mathrm{CPB}^{-C T E}$ in transfected COS-7 cells were carried out and qualified by detection of GFP expression via fluorescence microscopy at different time intervals $(24,48$ and $72 \mathrm{hrs}$ ) after transfection.

\section{Flow cytometry analysis}

At 48 hrs after incubation, cells in 4-well plates were washed once with $300 \mu \mathrm{l}$ of PBS and were detached with $300 \mu \mathrm{l}$ of trypsin/EDTA. Then the cells were centrifuged at $1500 \mathrm{~g}$ and the supernatant was discarded. The cells were resuspended in RPMI and kept on ice. Afterward, cells were directly applied to a Partec PASIII flow cytometer (Partec $\mathrm{GmbH}$, Germany) using a bivariate scatter plot of fluorescence versus side scatter, by gate setting with un-transfected cells. A minimum of 10,000 events from the viable transfected cell population per sample were analyzed.

\section{STATISTICAL ANALYSIS}

All of the pharmaceutical experiments were repeated 3 times and all measurements were repeated three to five times. Means and standard deviations were calculated using GraphPad Prism 5.0 (if necessary). The statistical analysis between different groups was determined with an ANOVA test. Differences were considered statistically significant when $p<0.05$. 


\section{RESULTS}

\section{Plasmid construction and purification}

The PCR products corresponding to the cpa (696 $\mathrm{bp}$ ), $\mathrm{c} p b\left(950 \mathrm{bp}\right.$ ) and $\mathrm{c} p b^{-C T E}$ (650 bp) genes, were successfully ligated into pEGFP-N1 and clones were confirmed by PCR and enzymatic digestion (data not shown). The obtained recombinant plasmids named as pEGFP-cpa, pEGFP-cpb and pEGFP- $c p b^{-C T E}$ were prepared by Qiagen plasmid extraction midi kit.

\section{cSLN Formulation and characterization}

Response analysis of the results from full factorial design (Table 1) demonstrated that a formulation with total lipid:Tween 80 ratio of 3.25 processed by $\mathrm{M}+\mathrm{H}$ method (S formulation) has a proper size of $257 \pm 21 \mathrm{~nm}$, zeta potential of $+52 \pm 6$, and size distribution of $0.34 \pm 0.08$. This formulation was stable for 30 days $(p<0.05)$. Formulations $\mathrm{S}_{1-9}$ were prepared and characterized according to their size and zeta potential and poly dispersity index and stability assessments after 30 days for (Table 2). Increasing homogenization time and rate did not show any significant decrease in the particle size. Results were also reproducible within two separate experiments. We utilize DNA surface adsorption via direct complexation of constant amounts $(5 \mu \mathrm{g})$ of each pDNA (pEGFP-cpa, pEGFP- $c p b$ and pEGFP- $c p b^{C T E}$ ) with cSLNs as a simple and highly efficient method. Table 3 presents the formulations according to their DOTAP: pDNA ratios and their characteristics.

As shown in Fig. 1, gel retardation assay for SLN-pDNA revealed different complexation strength in various DOTAP:pDNA ratios. Clearly unbound free pDNA bands were visible for $\mathrm{SD}_{6}$ and $\mathrm{SD}_{7}$ (Table 3) formulations (Fig. 1, Lanes 6 and 7). Thus a complete complexation between pDNA and SLN was achieved at a DOTAP:pDNA:ratio higher than 3:1(Lane 1-5) and either free or retarded pDNA bands were not visible in these samples.

\begin{tabular}{|c|c|c|c|c|c|c|}
\hline $\begin{array}{l}\text { Formulation } \\
\text { code }\end{array}$ & $\begin{array}{l}\text { Size }(\mathrm{nm}) \\
\text { Day } 0\end{array}$ & $\begin{array}{l}\text { PDI } \\
\text { Day } 0 \\
\end{array}$ & $\begin{array}{l}\text { Zeta potential } \\
\text { Day } 0\end{array}$ & $\begin{array}{l}\text { Size }(\mathrm{nm}) \\
\text { Day } 30 \\
\end{array}$ & $\begin{array}{l}\text { PDI } \\
\text { Day } 30 \\
\end{array}$ & $\begin{array}{l}\text { Zeta potential } \\
\text { Day } 30\end{array}$ \\
\hline $\mathrm{S}_{1}$ & 827 & 0.59 & -7.0 & 931 & 0.6 & -5.0 \\
\hline $\mathrm{S}_{2}$ & 1136 & 0.57 & -9.4 & 1215 & 0.61 & -8.7 \\
\hline $\mathrm{S}_{3}$ & 767 & 0.42 & -9.0 & 1132 & 0.59 & -8.3 \\
\hline $\mathrm{S}_{4}$ & 695 & 0.45 & -8.5 & 817 & 0.40 & -9.0 \\
\hline $\mathrm{S}_{5}$ & 419 & 0.5 & -9.2 & 648 & 0.50 & -8.0 \\
\hline $\mathrm{S}_{6}$ & 414 & 0.37 & -9.8 & 619 & 0.40 & -12.0 \\
\hline $\mathrm{S}_{7}$ & 235 & 0.52 & -10.0 & 279 & 0.63 & -10.0 \\
\hline $\mathrm{S}_{8}$ & 277 & 0.48 & -10.6 & 265 & 0.59 & -9.0 \\
\hline $\mathrm{S}_{9}$ & 253 & 0.2 & -18.24 & 286 & 0.32 & -18.0 \\
\hline
\end{tabular}

\begin{tabular}{|c|c|c|c|}
\hline $\begin{array}{l}\text { Formulation } \\
\text { code }\end{array}$ & DOTAP:pDNA Ratio & Size (nm) & Zeta (mv) \\
\hline $\mathrm{SD}_{1}$ & $9: 1$ & 185 & +46 \\
\hline $\mathrm{SD}_{2}$ & $7: 1$ & 197 & +42 \\
\hline $\mathrm{SD}_{3}$ & $5: 1$ & 218 & +37 \\
\hline $\mathrm{SD}_{4}$ & $4: 1$ & 245 & +23 \\
\hline $\mathrm{SD}_{5}$ & $3: 1$ & 646 & +22 \\
\hline $\mathrm{SD}_{6}$ & $2: 1$ & 1136 & -12 \\
\hline $\mathrm{SD}_{7}$ & $1: 1$ & 1179 & -17 \\
\hline $\mathrm{S}$ & - & 257 & +52 \\
\hline
\end{tabular}


$\mathrm{SD}_{1}$ and $\mathrm{SD}_{2}$ formulations were able to efficiently condense pDNAs. Therefore, ethidium bromide could not intercalate within the DNA chains, and no fluorescence was detectable clearly under UV light (Fig .1, Lanes 1 and 2).

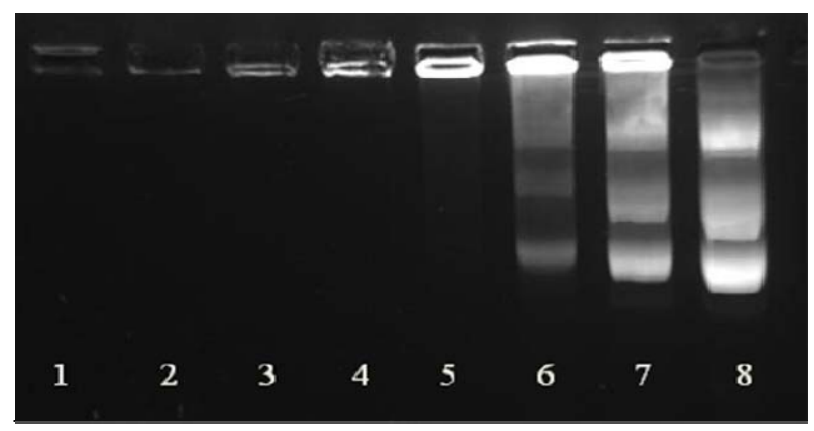

Figure 1. Gel retardation assay of cSLN-pEGFP-cpb with different DOTAP:pDNA ratios. Lanes 1-7 samples with formulation codes SD1-7 and lane 8 is free pDNA as control.

In addition, all of the formulations subjected to DNase I digestion and undigested pDNAs were recovered and visualized by agarose gel electrophoresis as described in Materials and Methods. The integrity of pDNAs was studied immediately after loading and before transfection studies and was compared to both untreated (Fig. 2, Lane 1) and treated (Fig. 2, Lane 2) control pDNAs. As shown in Fig. 2, naked pDNA was completely degraded under the experimental conditions (Fig. 2, Lane 2). Unbound free pDNAs bands were observed for $\mathrm{SD}_{1}$ formulation (Fig. 2, Lane 3) comparable with untreated intact DNA (Fig. 2, Lane 1), confirming the data obtained by the gel retardation assay. As it is demonstrated in Fig 2, the pDNAs in $\mathrm{SD}_{1-4}$ were completely protected from DNase I digestion (Fig. 2, Lanes 3-6) and the greater part of the released pDNA maintained its supercoiled conformation more similar to the control pDNA (Fig. 2, Lane 1). When the complex ratio was 3:1 and lower, both free and retarded pDNA bands were faintly visible (Fig. 2, Lanes 79), indicating that there has been no full complex formation as previously observed in the gel retardation assay. Reproducibility of this assay was confirmed at 3, 7 and 14 days after loading, on the formulations stored at $4^{\circ} \mathrm{C}$. The incubation time necessary for degrading $5 \mu \mathrm{g}$ of naked pDNA was determined to be $60 \mathrm{~min}$.

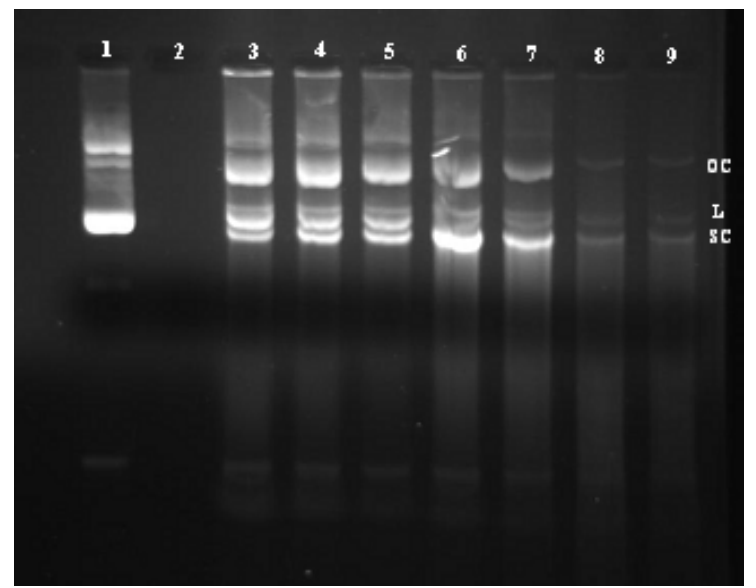

Figure 2. Agarose gel electrophoresis of pEGFP extracted from cSLN-pEGFP-cpb showing protection of pDNA from DNase I digestion. Lanes 1 and 2 correspond to control intact and DNase I-treated pDNA. Lanes 3-9 correspond to pDNA extracted from formulations $\mathrm{SD}{ }_{1-7}$, respectively. The lower band is supercoiled (S.C.) pDNA and the upper band is open circular (O.C.) pDNA.

The storage stability analysis of the fresh $\mathrm{SD}_{1-9}$ formulations and those stored in dark for up to 4 weeks at different temperatures $\left(4 \pm 1{ }^{\circ} \mathrm{C}\right.$ and $25 \pm$ $1^{\circ} \mathrm{C}$ ) revealed different sizes and zeta potentials due to the DOTAP percents. DNA-loaded SLNs, when stored at refrigerated temperature $\left(4 \pm 1^{\circ} \mathrm{C}\right)$, were more stable in comparison with room temperature storage (Fig. 3). No statistically significant differences $(p<0.05)$ were observed between the formulations prepared at different time points.

\section{Cytotoxicity evaluation of cSLN formulations}

In vitro toxicity of cSLN and PEI as well as naked pDNA was evaluated in parallel on COS-7 cells and determined as a function of formulation concentration, by using $\mathrm{SD}_{3,4}$ formulations and PEI in N/P ratios of 7, 10, and 13 all without plasmids. Both $\mathrm{SD}_{3,4}$ formulations were found to be non-toxic comparable to pDNA treated and none treated COS-7 cells, as shown in Fig. 4. There was a slight reduction in cell viabilities in $\mathrm{SD}_{3}$ formulations, but we did not observe a significant difference $(p<$ $0.05)$ in the viability of these formulations. Average cell viabilities were between 80 to $92 \%$ of untreated COS-7 cells as a negative control at different time points including 24, 48 and $72 \mathrm{hrs}$ after treating with $\mathrm{SD}_{4}$ formulation and $100 \%$ for the treated COS-7 cells with $5 \mu \mathrm{g}$ pDNA sample. 

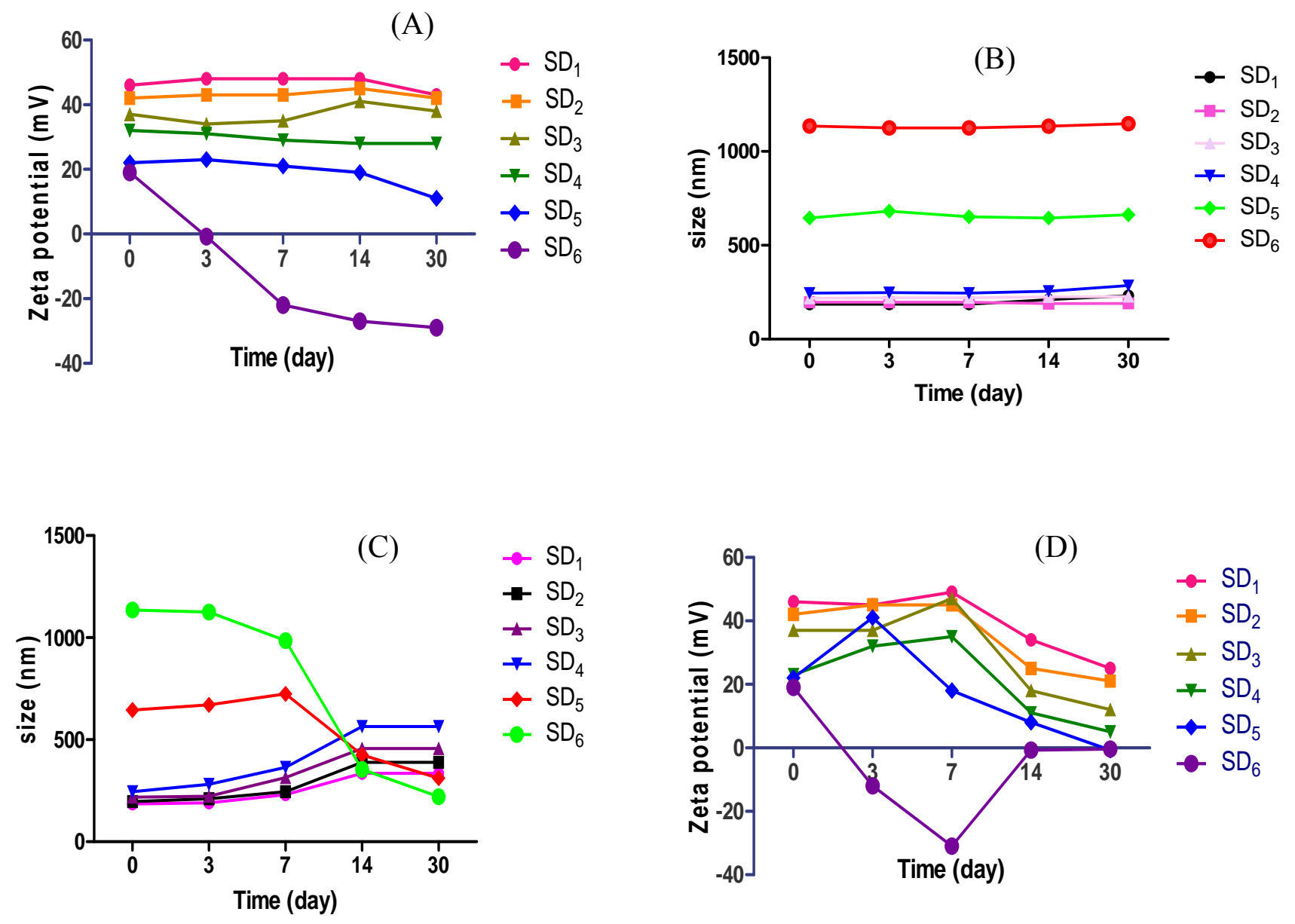

Figure 3. Zeta potential and size of cSLN-pEGFP-cpb. Zeta potentials at two different temperatures, stored at $4 \pm 1{ }^{\circ} \mathrm{C}(\mathrm{a})$ and room temperature (c). Sizes at two different temperatures, stored at $4 \pm 1{ }^{\circ} \mathrm{C}(\mathrm{b})$ and room temperature (d).

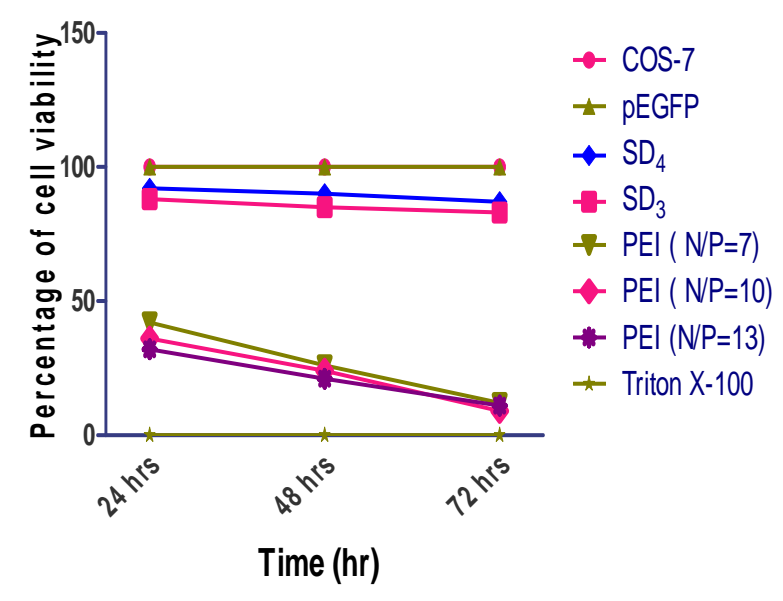

Figure 4. COS-7 cell viability after treating with $\mathrm{pEGFP}$, Triton $\mathrm{X}-100, \mathrm{SD}_{4,5}$ and $\mathrm{PEI}(\mathrm{N} / \mathrm{P}=7,10,13)$.
Cell viabilities were 26, 24 and $21 \%$ for PEI in the $\mathrm{N} / \mathrm{P}$ ratio of 7, 10, and 13; respectively. After $48 \mathrm{hrs}$ there was a significant reduction in cell viabilities while using PEI only.

Incubation of cells with the $\mathrm{SD}_{4}$ formulation loaded by pEGFP-cpa, pEGFP- $c p b$, pEGFP-cpb ${ }^{\text {-CTE }}$ retained $89-86 \%, 82-81 \%$ and $92-87 \%$ viability, respectively (Fig. 5). Similarly, the least cytotoxic PEI ratio $(\mathrm{N} / \mathrm{P}=7)$ used to deliver $\mathrm{pDNAs}$ revealed $52-33 \%, \quad 49-25 \%, \quad 59-21 \%$ from $24-72 \mathrm{hrs}$, respectively. Even when the transfection time was up to $72 \mathrm{hrs}$, the cytotoxicity of $\mathrm{SD}_{4}$ formulations didn't changed significantly $(p<0.05)$ but the cytotoxicity of PEI increased significantly after this time $(p<0.05)$. Additionally, pEGFP- $c p b$ expression, cell viability is at the lowest level. This difference is more significant $(p<0.05)$ when utilizing cSLN as the delivery carrier. 


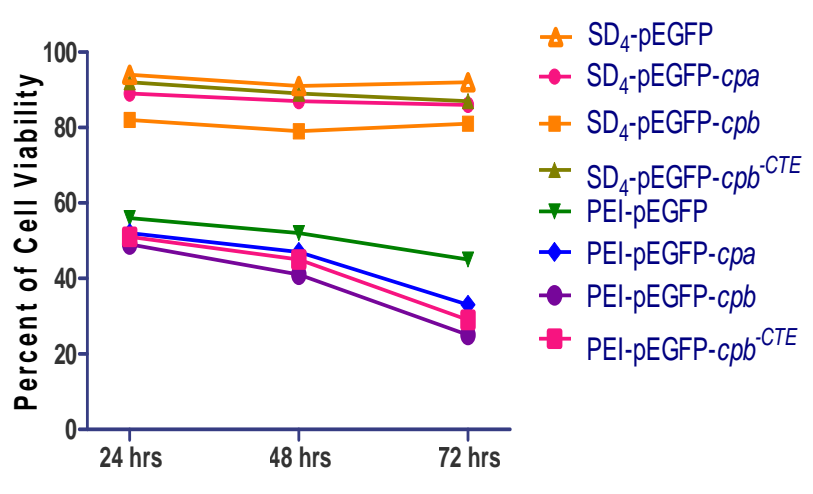

Figure 5. COS-7 cell viability after treating with $\mathrm{SD}_{4}$ pDNAs and PEI-pDNAs.

\section{Transfection analysis}

Microscopic observations revealed that $\mathrm{SD}_{4}$ formulation did not induce any significant alteration in the cell morphology compared to the negative control over the two concentrations range and different time tested. The MTT assay of $\mathrm{SD}_{4}$-pDNA formulation using different genes did not reveal similar toxicity results as shown in Fig. 5. This indicated that the related expressed proteins could affect the cell growth and this was more significant when formulating with cSLN as the less cytotoxic carrier compared to PEI. We observed higher expression of GFP in cells treated with PEI-pDNAs (Fig. 6). This effect was a general observation for all of the recombinant vectors (pEGFP-cpa, pEGFP- $c p b$, pEGFP-cpb ${ }^{-C T E}$ ). As a part of the experiment, we tested the influence of the transfection time on gene expression. Although higher transfection times ( 72 hours versus 24 and 48 hours) increased the protein expression levels, microscopic evaluation of the cell viability also showed more dead cells when using PEI as the delivery system. This observation was not due to nutrient depletion of the media as the cells in contact with $\mathrm{SD}_{4}$ formulation were still alive. This observation had concurrence with the MTT results after similar time intervals. We also studied the effect of transfection time on transfection efficiency by comparing the results in different transfection times $(24,48$ and $72 \mathrm{hrs})$. The efficiency of 48 and $72 \mathrm{hrs}$ transfection was not significantly different as we did not observe any change in transfection efficiency after $72 \mathrm{hrs}$ of storage. Due to the MTT viability results and the visually observed cell deformation and death when transfecting cells with PEI at longer incubation times, a 48-hour incubation time was selected for flow cytometry evaluation. The highest expression levels associated with the percent of cells expressing GFP (Fig. 6 and 7) was obtained in the case of PEI-pEGFP $(29.14 \%)$. This level was statistically higher $(p<0.05)$ than the percentage of transfected cells obtained with the $\mathrm{SD}_{4}$-pEGFP (14.64\%). No statistically significant differences in the cells expressing GFP transfected with $\mathrm{SD}_{4}$-pEGF-cpa and $\mathrm{SD}_{4}$-pEGF- $c p b^{-C T E}$ were found. However, GFP expression was significantly higher in PEI-pEGF$c p a$ and PEI-pEGF- $c p b^{-C T E}(p>0.05)$ (Fig. 7).

\section{Determination of efficiency/toxicity ratio}

Efficiency/toxicity factor was determined as a ratio of expression percent to toxicity percent obtained from flow cytometry and MTT tests, respectively. Fig. 8 presents these ratios according to the carriers and genes to be delivered. As shown in the figure, the efficiency/toxicity ratio of SD4 as a delivery carrier is twofold more than that of PEI when harboring the pEGFP-N1 without any inserted genes. In addition, for $\mathrm{pEGF}-\mathrm{cpb}$ delivery which is happened to express the most cytotoxic $\mathrm{CP}$ amongst the evaluated genes (Fig. 5), this factor showed no significant difference using either of these carriers.

\section{DISCUSSIONS}

Recovery and protection in leishmaniasis at least in animal models is associated with development of Th1 type of immune response (17). The most important step in developing an effective vaccine against leishmaniasis is to identify appropriate antigens, delivery systems (adjuvants) and to have the knowledge of the type of immune response generated in protected hosts. Previously, we have reported constructing eukaryotic plasmids harboring $c p a$ and $c p b$ and demonstrated that a cocktail vaccine containing these genes is possible and effective for leishmaniasis (8). However, recombinant naked pDNA is not proven as a powerful tool for DNA vaccination because of its rapid elimination from the circulation. 


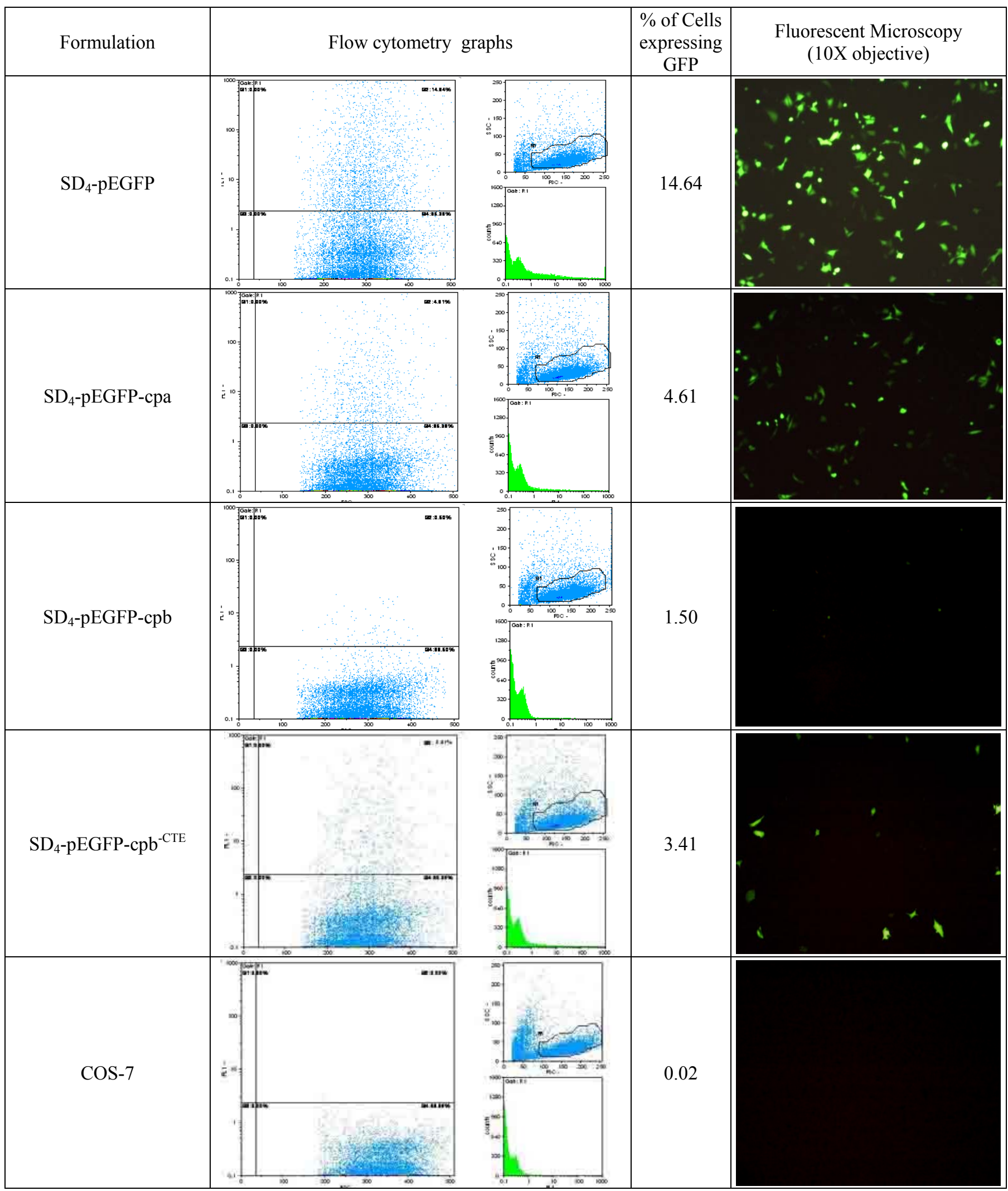




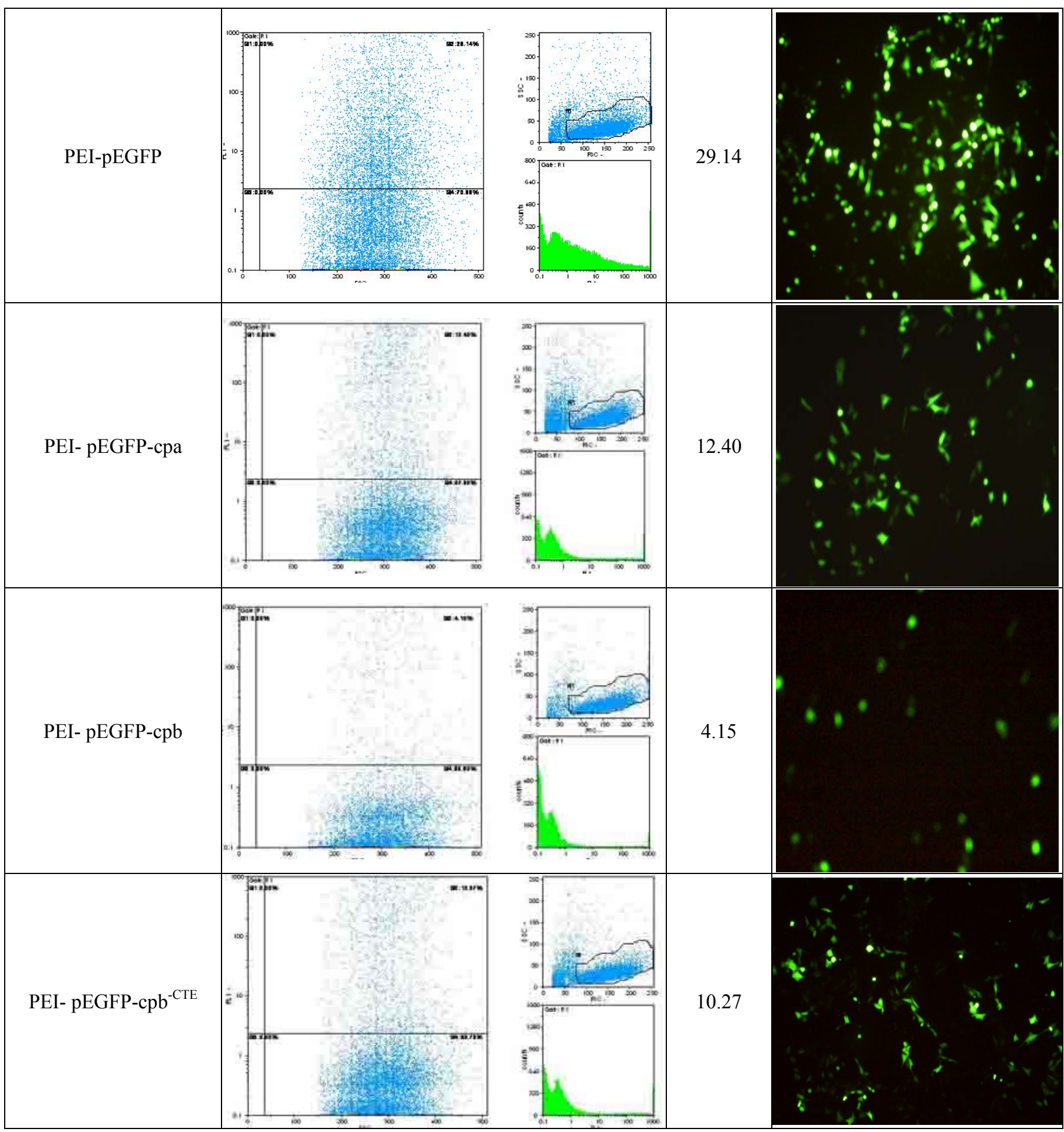

Figure 6. GFP-positive cells obtained with $\mathrm{SD}_{4}-\mathrm{pDNA}$ complexes and PEI-pDNA (N/P=7) complexes observed by inverted fluorescent microscopy (10X objective) and the related flow cytometry and efficieny/toxicity ratios assessment at 48 hrs. 


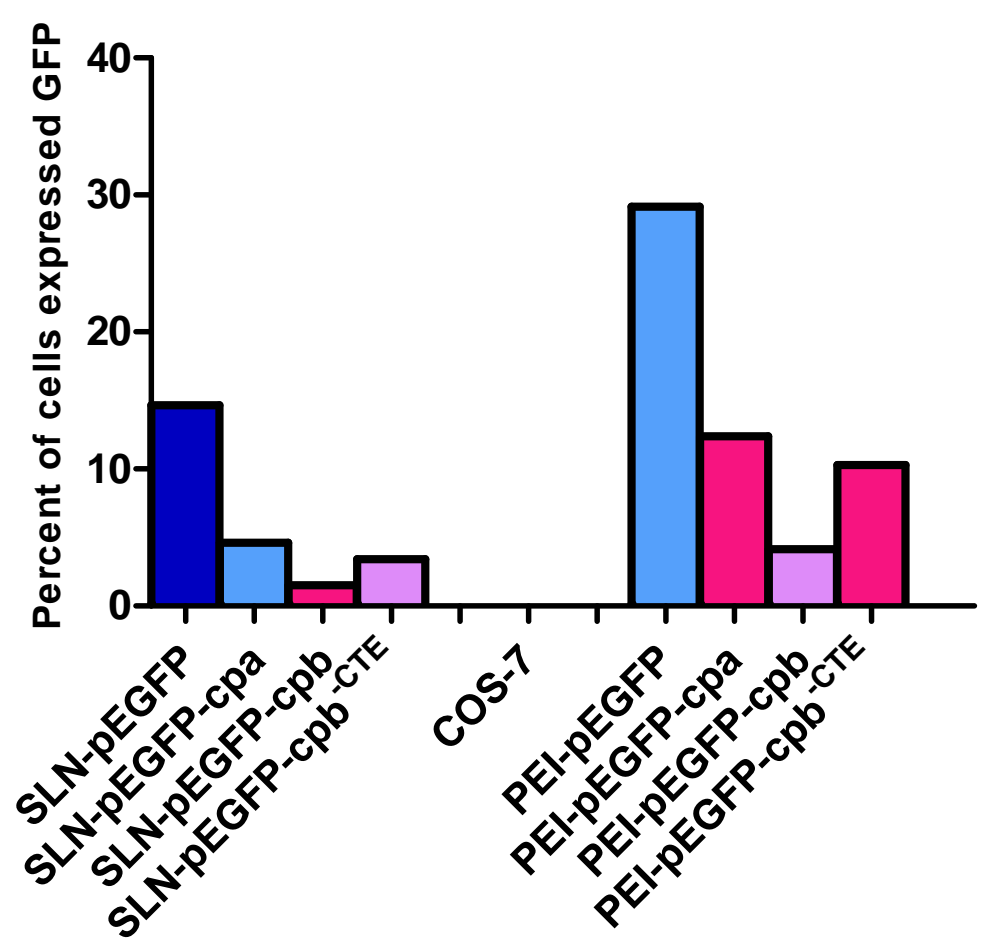

Figure 7. Percentage of GFP-expressing cells determined by flow cytometry after treating cells with $\mathrm{SD}_{4}-\mathrm{pDNA}^{-}$ complexes $(\mathrm{w} / \mathrm{w})$ and PEI-pDNA complexes $(\mathrm{N} / \mathrm{P}=7)$.

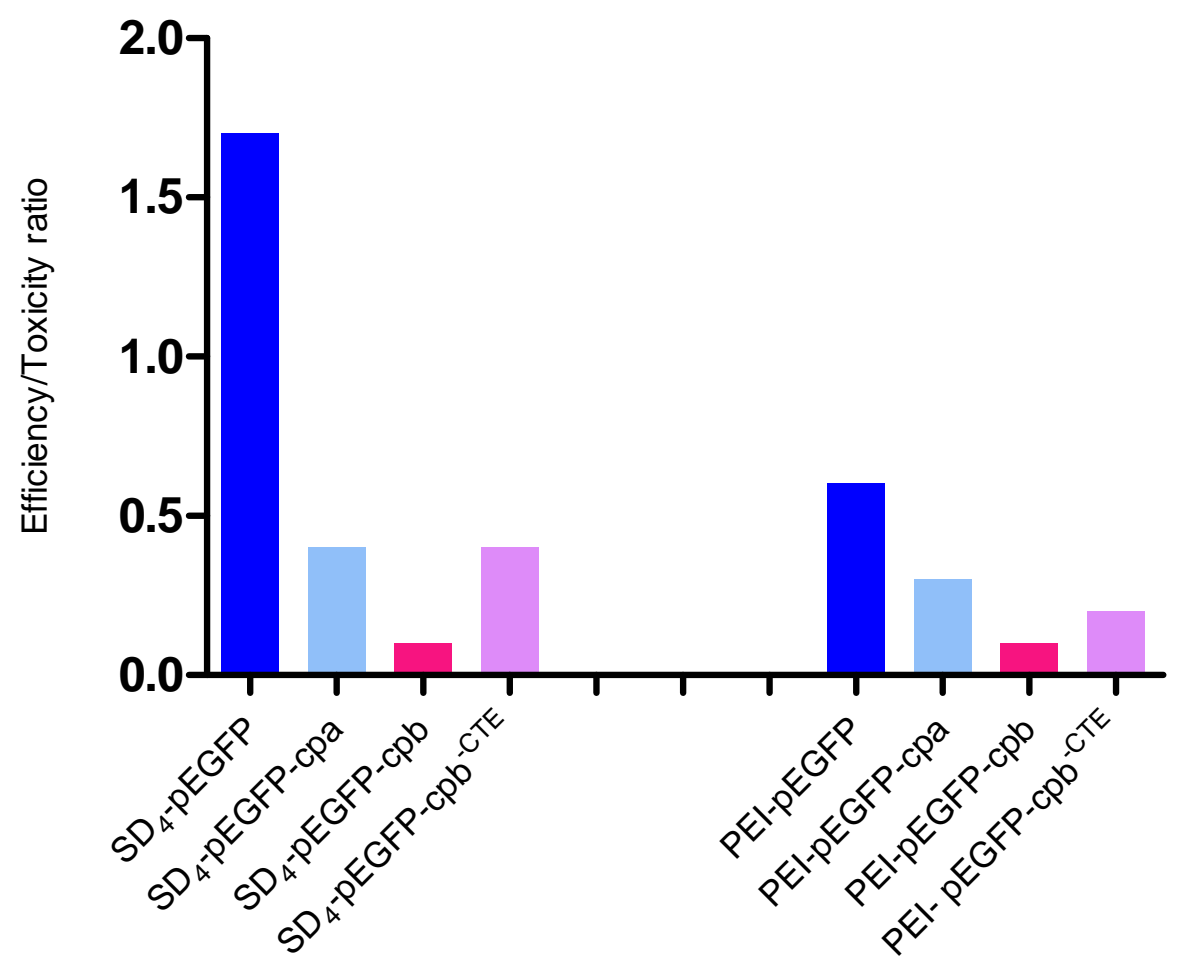

Figure 8. Efficiency/toxicity ratio of the carriers loaded with different genes 
In the present study, the efficiency of cSLNs as a delivery system to transfect COS-7 cells were investigated for further in vivo vaccine studies and protection against challenge infection. As a carrier system, solid lipid nanoparticles have many features that are valuable for nanomedicine. So far, SLNs have been investigated as delivery systems mostly for drugs and rarely for genes (14-16, 18-20). In both cases, this delivery system is reported to proceed more efficiently compared to the drug or gene alone. cSLN has been shown to condense DNA into nanometric colloidal particles capable of transfecting mammalian cells in vitro $(14,15,18$, 20). Very recently, cSLN application for gene delivery has been conveniently investigated showing ability to induce the expression of a foreign protein in vivo (16). This supports the potential of SLNs for gene and vaccine delivery $(16,23,24)$. cSLN offers several technological advantages when compared with standard DNA carriers such as cationic lipids or polymers, e.g. relatively easy production and in most cases without utilizing any organic solvent, large scale production possibility in qualified production lines, good storage stabilities, possibility of steam sterilization and lyophilization $(11,20,23)$.

Hence, we aimed to evaluate cSLNs ability for in vitro delivery of $c p a, c p b$ and $c p b^{\mathrm{CTE}}$ intended to be used as a cocktail DNA vaccine in forthcoming studies on animal models to cover the main drawback of naked pDNA, that is rapid elimination from the circulation when used for DNA vaccination. In this regard, formulation $\mathrm{S}$ with a proper size and stability was prepared. The nanoparticles formulated of cetyl palmitate, cholesterol, DOTAP and Tween 80, and their transfection capacity and cytotoxicity were evaluated and compared to PEI $25 \mathrm{kDa}$, a highly efficient cationic polymer (21). Furthermore, we evaluated the influence of the DOTAP/DNA ratio on the protection ability and cytotoxicity of the SLN-DNA complexes.

In our formulation process, there were at least three key steps. The first one was adjusting the amount of the surfactant for the required amount of core and cationic lipid (totally 5.1\%). The second was utilizing the proper available method of preparation in our laboratory, i.e. homogenizing the coarse obtained $\mathrm{o} / \mathrm{w}$ emulsion with high shear that resulted in a fine emulsion. Consequently, we can claim that melt emulsification followed by homogenization of the lipid phase after adding hot aqueous DOTAP solution for $15 \mathrm{~min}$ is a feasible and reproducible method which appears to be sufficient for producing a fine emulsion with average particle size of $257 \mathrm{~nm}$. We used this method for further investigations. The last key step was anchoring pDNA on the surface of cSLNs. The SLN-pDNA complexes were prepared immediately after preparation of S formulation. One of the major negative aspects of the DNA encapsulated formulations is the exposure of pDNA to harsh conditions during particle preparation that causes DNA damage and integrity loss. To avoid this, we utilized a surface adsorption method via direct complexation of a constant amount of pDNA $(5 \mu \mathrm{g})$ with different weight ratios of DOTAP in formulation S. cSLN-pDNA complexes $\left(\mathrm{S}_{1-9}\right.$ formulations) were characterized and evaluated regarding size, zeta potential, nuclease protection, in vitro transfection efficiency, and cell viability.

As demonstrated in Table $3, \mathrm{SD}_{1-4}$ formulations had smaller size than $\mathrm{S}$ formulation. These formulations correspond to DOTAP:pDNA ratios from $9: 1$ to $4: 1(185 \mathrm{~nm}$ to $245 \mathrm{~nm})$. The size reduction and degree of pDNA condensation with regards to increasing the cationic lipid:pDNA ratio were comparable to the results reported by del Pozo-Rodríguez (15). The zeta potentials (Table 3) were positive (between +46 to $+23 \mathrm{mV}$ ) for these DOTAP:pDNA ratios. For transfection purposes, these positive charges are helpful for supporting the interaction of cSLN delivery system with negatively charged COS-7 cell membrane and thus cell entry. However the transfection levels reported by del Pozo-Rodríguez was slightly smaller than we are reporting here (15). This must be due to the presence of cholesterol domains in our cSLN formulation which enhances transfection by facilitating membrane fusion.

The inherent instability observed for the cSLN suspension is a severe limitation for the practical application of these carriers in medicine. Therefore, in order to obtain efficient vectors, we studied the cSLNs-pDNA formulations stability and observed that $\mathrm{SD}_{1-4}$ formulations can be kept in $4^{\circ} \mathrm{C}$ up to 30 days. It is worth mentioning that we did not observe any significant $(p<0.05)$ change in size and zeta potential during this storage period. This means there was no tendency to loose pDNA and agglomerate during storage at $4^{\circ} \mathrm{C}$. Therefore, for at least one month after storage at $4^{\circ} \mathrm{C}$, constant 
transfection efficiency would be expected. It is highly probable that the presence of Tween 80 as an additive in cSLN formulation enhance its physical stability. During stability studies, protection ability of these formulations for the adsorbed pDNAs was tested by DNase I challenge assay. Our results suggest that the examined $\mathrm{SD}_{1-4}$ formulations are expected to be stable in the presence of serum nucleases and cell endonucleases, being able to deliver pDNA in a functionally active form with good structural integrity.

According to the size, zeta potential, DNase I assay and stability study results, $\mathrm{SD}_{3,4}$ formulations were selected for further tests like determining cytotoxicity. In addition, according to the size and zeta potentials (Table 3 ), it was possible to obtain high percentage of fluorescent cells using $\mathrm{SD}_{1-3}$ formulations. However, an increase in the cationic lipid ratio in $\mathrm{SD}_{3}$ formulation resulted in lower cell viability. The $\mathrm{SD}_{4}$ formulation, which revealed acceptable size, zeta, storage ability and minor cytotoxicity (87-92\% in $72 \mathrm{hrs}$ ) were used in the next step. $\mathrm{SD}_{4}$ formulation (less cytotoxic carrier) in complex with pDNAs harboring different genes demonstrated significantly different cytotoxicity patterns. This means that cytotoxic effect of the proteins which are going to be expressed is more noticeable when utilizing SLN as the less cytotoxic delivery carrier. Conversely, this difference is lower when being formulated with PEI (more cytotoxic carrier). This observation might be due to the higher cytotoxicity of PEI that will mask the cytotoxic properties of a gene that is going to be expressed after gene delivery to the nucleus.

It is worth mentioning that using substances that are generally recognized as safe (GRAS) with suitable amounts in $\mathrm{SD}_{4}$ formulation lead to an advantageous toxicity profile when compared with polyethylenimine (PEI). This cationic polymer may cause liver necrosis and animal death after intravenous application and induces neutrophil infiltration and reduced lung function upon intratracheal application (20). However, this carrier is still considered as a gold standard for efficient gene delivery, at least for in vitro application. Accordingly, to make a judgment between these two delivery systems (i.e. SLN and PEI) by means of their potency to transfect cells in vitro, the transfected cells were analyzed by flow cytometry as well as fluorescence microscopy and the related cytotoxicities were evaluated by MTT test as well.
We observed higher percentage of GFP-expressing cells in cells treated with PEI-pDNAs, qualitatively and quantitatively. The explanation is that uncomplicated cationic particles have a residual positive charge after adsorption of pDNA, which leads to attachment of nanoparticles onto the surface of cells (21). The amount of residual charge present on the nanoparticles can determine their ability to stick to the cell membrane and consequently be taken up by the cells. Complexes prepared with PEI may have a higher amount of residual positive charge after loading of pDNA, due to their higher initial zeta potential which may facilitate efficient cellular internalization of nanoparticles. Higher zeta potential coupled with smaller size may be responsible for higher transfection efficiency of PEI-pDNA. This data is in agreement with other reports that stated in the absence of endosomolytic agents such as chloroquine, gene transfer efficiency mediated by SLN-derived gene vectors is lower than PEI $25 \mathrm{kDa}$ $(14,18,20)$.

Our results showed the ability of $\mathrm{SD}_{4}$ formulation containing Cysteine proteinase genes to transfect COS-7 cells and we were able to detect the green fluorescent protein quantifiably by fluorescent microscopy and also quantitatively by flow cytometry, although transfection efficiencies were lower than of PEI. Here, we point at an important feature of a non-viral transfection system, the efficiency/toxicity, which is determined as a ratio of pDNA expression percent to toxicity percent obtained from MTT test. Considering this feature, even though the $\mathrm{SD}_{4}$ transfection efficiency was lower than that of the commercially available PEI, it is much better tolerated by the cells, having lower cytotoxicity confirmed by MTT results. Therefore, rational practical decision can be made only by comparing efficiency/toxicity ratio when in vivo utility is considered. As our data indicates, the efficiency/toxicity ratio of $\mathrm{SD}_{4}$ as a delivery carrier is twofold more than that of PEI when harboring the pEGFP-N1 without any inserted genes. This was owed to the vigilant selection of nontoxic and nonirritant materials while formulating cSLNs. Our results further confirmed the data describing that cSLN composed of Precirol ${ }^{\circledR}$ ATO 5, DOTAP and Tween 80 had adequate transfection stability and did not show any decrease in cell viability and stability properties even after one month storage at $4{ }^{\circ} \mathrm{C} \quad$ (15). We observed that related 
efficiency/cytotoxicity ratio of carriers is not only dependent on the delivery system but also depends to the gene which is going to be expressed. The difference between delivery systems was minimized when viability of cells decreased after expression of the associated genes. This was observed with $\mathrm{CPB}$ expression, which happened to be the most cytotoxic protein between the cysteine proteinases tested here. Therefore, we suggest that for such a more cytotoxic pDNA, a carrier with lower cytotoxicity would be more efficient to be utilized.

In summary, in the present study, cSLNspDNA formulations harboring Cysteine proteinase genes were prepared and characterized regarding size, zeta potential and nuclease protection. In vitro transfection efficiency and cell viability were also evaluated and compared to linear PEI $25 \mathrm{kDa}$, the most powerful non-viral gene delivery carrier. cSLNs have demonstrated transfection capacity in vitro as confirmed by previous studies $(14,15,18$, 23 , 24). Being relatively cost-effective, biodegradable, non-toxic and stable, $\mathrm{SD}_{4}$ formulation seems to have a good prospect as an effective delivery carrier for cocktail DNA immunization for leishmaniasis at least in animals. Gel retardation assays and GFP protein expression confirmed by flow cytometry proved that the positive charge of $\mathrm{SD}_{4}$-pDNAs $(\zeta$ potential $=+23 \mathrm{mV}$ ) was suitable for interaction with the negatively charged cell surface and the cell entry. In addition, $\mathrm{SD}_{4}$ formulation could compact pDNAs, thus prevent plasmid DNA degradation induced by DNas I enzyme. This formulation also showed CPs expression capacity in vitro at least for $72 \mathrm{hrs}$ after their COS-7 cells treatment without a significant reduction in cell viability.

\section{CONCLUSIONS}

During the last decades, vaccine delivery has made considerable progress although this field is still confronted with several challenges, mainly concerning antigen immunogenicity, delivery and stability. This groundwork study shows the capacity of $\mathrm{SD}_{4}$-pDNA formulation to drive the expression of these genes while covering the most concerned criteria for pDNA delivery carriers that is cytotoxicity. Cocktail DNA vaccine and in vivo immunization against leishmaniasis is currently underway in our laboratory. Considering that SLN marketing arrival might not be too far (13), and assuming this formulation will promote cps delivery, this strategy may provide a suitable vaccine platform against leishmaniasis. From a practical point of view, this work demonstrated joint efforts of two different disciplines involved that are needed to meet vaccine design challenges.

\section{ACKNOWLEDGEMENTS}

D. Doroud thanks Pasteur Institute of Iran and Tehran University of Medical sciences for supporting her PhD studentship. The authors thank Dr. M. Amanzadeh, Cell Bank of Pasteur Institute of Iran, for his advice and guidance on the MTT assay and Dr. A. Saadat and Dr. E. Moazeni, Pharmaceutics Dept., Faculty of Pharmacy, Tehran University of Medical Sciences, for their technical support on size and zeta potential determinations. We are also grateful for English revision by Amir Mizbani.

\section{REFERENCES}

1. WHO. Leishmaniasis Home Page. World Health Organization website, 8-21-2006, http://www.who.int/leishmaniasis/burden/en/.

2. Kedzierski, L., Sakthianandeswaren, A., Curtis, JM., Andrews, PC., Junk, PC. and Kedzierska, K., Leishmaniasis: current treatment and prospects for new drugs and vaccines. Curr Med Chem., 16:599614. 2009.

3. Verthelyi, D. and Klinman, DM., Immunoregulatory activity of $\mathrm{CpG}$ oligonucleotides in humans and nonhuman primates. Clin Immunol, 109:64-71, 2003.

4. Reed, S. G., Bertholet, S., Coler, R. N. and Friede, M., New horizons in adjuvants for vaccine development . Trends Immunol, 30: 23-32, 2009.

5. Palatnik-de-Sousa, C. B., Vaccines for leishmaniasis in the fore coming 25 years. Vaccine, 26: 17091724, 2008.

6. Rafati, S., Fasel, N. and Masina, S., Leishmania Cysteine Proteinases: From Gene to Subunit Vaccine. Curr Genomics, 4: 253-261. 2003.

7. Rafati, S., Salmanian, AH., Taheri, T., Vafa, M. And Fasel, N., A protective cocktail vaccine against murine cutaneous leishmaniasis with DNA encoding cysteine proteinases of Leishmania major. Vaccine. 19: 3369-75. 2001.

8. Rafati, S., Nakhaee, A., Taheri, T., Taslimi, Y., Darabi, H., Eravani, D., Sanos, S., Kaye, P., Taghikhani, M., Jamshidi, S. and Rad, MA., Protective vaccination against experimental canine visceral leishmaniasis using a combination of DNA 
and protein immunization with cysteine proteinases type I and II of L. infantum. Vaccine. 23: 3716-25. 2005.

9. Rafati, S., Zahedifard, F., Azari, M.K, Taslimi, Y. and Taheri, T., Leishmania infantum: prime boost vaccination with $\mathrm{C}$-terminal extension of cysteine proteinase type I displays both type 1 and 2 immune signatures in BALB/c mice. Exp Parasitol. 118: 393401, 2008.

10. Wilson, K.D., de Jong, SD. and Tam, YK., Lipidbased delivery of $\mathrm{CpG}$ oligonucleotides enhances immunotherapeutic efficacy. Adv Drug Del Rev, 61: 233-242, 2009.

11. Muller, R.H., Mader, K. and Gohla, S., Solid lipid nanoparticles (SLN) for controlled drug delivery-a review of the state of the art. Eur J Pharm Biopharm, 50: 161-177, 2000.

12. Muller, R.H., Ruhl, D., Runge, S., Schulze-Forster, K. and Mehnert, W., Cytotoxicity of solid lipid nanoparticles as a function of the lipid matrix and the surfactant. Pharm Res. 14: 458-462. 1997.

13. Joshi, M.D. and Müller, R.H., Lipid nanoparticles for parenteral delivery of actives. Eur $\mathrm{J}$ Phar Biopharm, 71:161-172, 2009.

14. Tabatt, K., Sameti, M., Olbrich, C., Müller, R.H. and Lehr, C.M., Effect of cationic lipid and matrix lipid composition on solid lipid nanoparticle-mediated gene transfer. Eur J Pharm Biopharm, 57: 155-162, 2004.

15. del Pozo-Rodríguez, A., Delgado, D., Solinís, M.A., Gascón, A.R. and Pedraz, J.L., Solid lipid nanoparticles: Formulation factors affecting cell transfection capacity. Int J Pharm, 339: 261-268, 2007.

16. del Pozo-Rodríguez, A., Delgado, D., Solinís, M. Á., Pedraz, J. L., Echevarría, E., Rodríguez, J. M. and Gascón, A. R., Solid lipid nanoparticles as potential tools for gene therapy: In vivo protein expression after intravenous administration. Int J Pharm., In Press, Corrected Proof, Available online 14 October 2009.

17. von Stebut, E. and Udey, M.C., Requirements for Th1-dependent immunity against infection with L. major. Microbs and Infection, 6:1102-1109, 2004.

18. Olbrich, C., Bakowsky, U., Lehr, C.M., Müller, R.H. and Kneuer, C., Cationic solid-lipid nanoparticles can efficiently bind and transfect plasmid DNA. J Control Rel, 77: 345-355. 2001.

19. Heiati, H., Tawashi, R. and Phillips, N.C., Drug retention and stability of solid lipid nanoparticles containing azidothymidine palmitate after autoclaving, storage and lyophilization. J Microencapsul, 15: 173-184, 1998.

20. Rudolph, C., Schillinger, U., Ortiz, A. Tabatt, K. Plank, C. and Muller, R.H., Application of novel solid lipid nanoparticle (SLN)-gene vector formulations based on a dimeric HIV-1 TAT-peptide in vitro and in vivo, Pharm Res, 21: 1662-1669, 2004.

21. Gebhart, C. L. and Kabanov, A.V., Evaluation of polyplexes as gene transfer agents. J Control Rel, 73: 401-416, 2001.

22. Feng, M., Lee, D. and Li, P., Intracellular uptake and release of poly (ethyleneimine)-co-poly (methyl methacrylate) nanoparticle/pDNA complexes for gene delivery. Int J Pharm., 311: 209-214, 2006.

23. Vighi, E., Ruozi, B., Montanari, M., Battini, R. and Leo, E., Re-dispersible cationic solid lipid nanoparticles (SLNs) freeze-dried without cryoprotectors: characterization and ability to bind the pEGFP-plasmid. Eur J Pharm Biopharm., 67(2):320-8, 2007.

24. Vighi, E., Ruozi, B., Montanari, M., Battini, R. and Leo, E., pDNA condensation capacity and in vitro gene delivery properties of cationic solid lipid nanoparticles. Int J Pharm, 389: 254-261, 2010. 